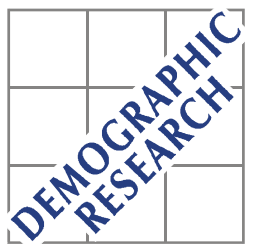

Demographic Research a free, expedited, online journal of peer-reviewed research and commentary in the population sciences published by the Max Planck Institute for Demographic Research Konrad-Zuse Str. 1, D-18057 Rostock · GERMANY www.demographic-research.org

DEMOGRAPHIC RESEARCH

VOLUME 13, ARTICLE 5, PAGES 117-142

PUBLISHED 05 OCTOBER 2005

http://www.demographic-research.org/Volumes/Vol13/5/

DOI: $10.4054 /$ DemRes.2005.13.5

Research Article

Changing mortality and average cohort life expectancy

Robert Schoen

Vladimir Canudas-Romo

(C) 2005 Max-Planck-Gesellschaft. 


\section{Table of Contents}

1 Introduction $\quad 118$

2 Aggregate measures of mortality 118

3 ACLE: A new measure of longevity 120

$4 \quad$ Cohort and period models of changing mortality 122

$5 \quad A C L E$ in England and Wales, Norway, and Switzerland 131

$\begin{array}{lll}6 & \text { Discussion and conclusions } & 135\end{array}$

$7 \quad$ Acknowledgments 136

$\begin{array}{ll}\text { References } & 137\end{array}$

Appendix: Decomposing the change over time in ACLE 139 


\title{
Changing mortality and average cohort life expectancy
}

\author{
Robert Schoen ${ }^{1}$ \\ Vladimir Canudas-Romo ${ }^{2}$
}

\begin{abstract}
Period life expectancy varies with changes in mortality, and should not be confused with the life expectancy of those alive during that period. Given past and likely future mortality changes, a recent debate has arisen on the usefulness of the period life expectancy as the leading measure of survivorship. An alternative aggregate measure of period mortality which has been seen as less sensitive to period changes, the crosssectional average length of life $(C A L)$ has been proposed as an alternative, but has received only limited empirical or analytical examination. Here, we introduce a new measure, the average cohort life expectancy $(A C L E)$, to provide a precise measure of the average length of life of cohorts alive at a given time. To compare the performance of $A C L E$ with $C A L$ and with period and cohort life expectancy, we first use population models with changing mortality. Then the four aggregate measures of mortality are calculated for England and Wales, Norway, and Switzerland for the years 1880 to 2000. $C A L$ is found to be sensitive to past and present changes in death rates. ACLE requires the most data, but gives the best representation of the survivorship of cohorts present at a given time.
\end{abstract}

\footnotetext{
${ }^{1}$ Department of Sociology, Pennsylvania State University, University Park, PA 16802; e-mail: schoen@pop.psu.edu

${ }^{2}$ Population Research Institute, Pennsylvania State University, University Park, PA 16802; e-mail: canudas@pop.psu.edu
} 


\section{Introduction}

A life table describes the mortality of a hypothetical birth cohort, and can summarize that experience in terms of life expectancy at birth (LE), i.e. the average number of years lived by the members of the life table cohort. When a life table is based on cohort data, it can reflect the survivorship of an actual group of people. In most cases however, life tables are based on mortality rates observed in a given year (or period). The period life expectancy from such a table can reflect the long term implications of recent behavior, but does not relate to the experience of any real cohort, and typically varies from year to year as death rates change. The question addressed here is "What is the average life expectancy of cohorts alive in a given year?" It is neither the period life expectancy nor the cohort life expectancy, and indeed is a quantity that has not previously been given a precise definition.

Recently a significant paper by Bongaarts and Feeney (2002) opened a new debate on how to interpret period life expectancy when rates of death vary over time. They argue that the period LE exhibits a tempo bias when "a rising (falling) mean age of persons at the occurrence of an event results in a temporary decline (increase) in numbers of events during the period of change" (p. 20). To correct for that bias, Bongaarts and Feeney (2002) advance an alternative measure that, in other work (Brouard 1986; Guillot 2003a), has been termed the cross-sectional average length of life ( $C A L$, a translation from the French durée de vie moyenne actuelle).

Vaupel (2002) and Guillot (2003b) have criticized that approach, citing its ambiguous definition of mortality tempo effects. Yet there is no doubt that the period LE can be seriously misleading if it is interpreted as the life expectancy of any actual group of persons. Here we do not attempt to adjust for tempo effects, but seek to advance the area by proposing a new measure that gives the average lifetime of a period population. Before presenting that measure, we discuss existing summary measures at greater length.

\section{Aggregate measures of mortality}

The most commonly known measures of mortality are the cohort and period life expectancy. Period life expectancy at age $a$ and time $t$ is calculated as the person-years lived above age $a$ divided by the number surviving from birth to age $a$. For example, the period life expectancy at birth at time $t$ can be expressed as 


$$
e_{p}(0, t)=\frac{\int_{0}^{\omega} \ell_{p}(a, t) d a}{\ell_{p}(0, t)},
$$

where $\ell_{p}(a, t)$ is the period survivorship function at age $a$ at time $t$ and $\omega$ is the highest age attained. Letting the radix of the table be one, i.e. $\ell_{p}(0, t)=1$, we see that $\ell_{p}(a, t)$ is the period life table probability of surviving from birth to age $a$. Denoting the force of mortality at age $a$ and time $t$ by $\mu(a, t)$, we can write the life table probability of surviving from birth to age $a$ as

$$
\ell_{p}(a, t)=\exp \left(-\int_{0}^{a} \mu(x, t) d x\right) .
$$

The subscript $p$ in equations (1) and (2) is used to denote period measures. In the rest of the text, subscript $c$ will be used to identify cohort measures. For example, $\ell_{c}(a, t-a)$ denotes the life table probability of surviving from birth to age $a$ for the cohort born at time $t$ - $a$, i.e.

$$
\ell_{c}(a, t-a)=\exp \left(-\int_{0}^{a} \mu(x, t-a+x) d x\right) .
$$

That cohort's life expectancy at birth, $e_{c}(0, t-a)$ is given by

$$
e_{c}(0, t-a)=\frac{\int_{0}^{\omega} \ell_{c}(x, t-a) d x}{\ell_{c}(0, t-a)},
$$

Since at exact age $a$ and time $t$ the cohort and period forces of mortality are identical, $\mu(a, t)$ does not have a subscript.

The cross-sectional average length of life, $C A L$, incorporates the past and present mortality of all active cohorts. Consider a population that has a constant number of births every year, but where mortality is free to vary over age and time. The number of persons in that population at time $\mathrm{t}$ is $C A L(t)$. Mathematically, it can be expressed as 


$$
C A L(t)=\int_{0}^{\omega} \ell_{c}(a, t-a) d a
$$

In the stationary population of the life table, with its constant age-specific mortality, $C A L$ equals life expectancy. In general, however, the size of $C A L(t)$ differs from a population's period LE at time t. Because in most contemporary populations mortality in past periods is generally greater than mortality at recent time $t$, the survival probabilities that are summed to produce $C A L(t)$ are usually lower than those that are summed to yield the period LE. As a result, the size of $C A L$ typically understates the current level of period mortality, that is the level reflected by the period LE.

\section{ACLE: A new measure of longevity}

At its core, the criticism of period life expectancy is that it fails to provide an accurate measure of the actual longevity of the persons in the population being examined. At present, however, no measure in demography does that, including $C A L$ (which does not incorporate mortality after the specified period). Here we fill that gap by presenting a new measure that does capture the average LE of active cohorts.

Our approach is straightforward: we specify a weighted average of the life expectancies at birth of all active cohorts. Weights are needed because it is not reasonable to give equal emphasis to newborns and to the few who survive to high ages. At every age, the weight we propose is the actual cohort probability of survival to that age. In effect, we let cohort survivorship provide the weights for our measure of average cohort survival.

Let $A C L E(t)$ denote this Average Cohort Life Expectancy. We can then write

$$
\operatorname{ACLE}(t)=\frac{\int_{0}^{\omega} e_{c}(0, t-a) \ell_{c}(a, t-a) d a}{\int_{0}^{\omega} \ell_{c}(a, t-a) d a}=\frac{\int_{0}^{\omega} e_{c}(0, t-a) \ell_{c}(a, t-a) d a}{C A L(t)},
$$

or

$$
A C L E(t)=\int_{0}^{\omega} e_{c}(0, t-a) C_{C A L}(a, t-a) d a,
$$


where $C_{C A L}(a, t-a)$ denotes the density distribution of the cohort survival probabilities, $C_{C A L}(a, t-a)=\frac{\ell_{c}(a, t-a)}{C A L(t)}$.

Bongaarts (2004) defined an alternative measure $A C L E_{p}$, using period deaths instead of cohort survivors as weights. Such a measure is likely to yield values quite different from $A C L E$, and will in some (though not all) cases approximate $C A L$. There are two reasons why we believe that alternative is not attractive. First, it seems much more logical to weight a measure of cohort survivorship by cohort survivors rather than by deaths. Second, some populations may have deaths occurring only at several high ages. If deaths were used as weights, only those few older cohorts would determine average cohort survivorship, defeating the purpose of having a measure that reflects the longevity of all living cohorts.

The value of our $A C L E(t)$ is the average of the life expectancies of the cohorts alive in year $t$, with those expectancies weighted by the $C A L$ population. When mortality is constant over time, period life expectancy, cohort life expectancy, ACLE, and $C A L$ are all equal. When mortality is declining, $A C L E$ will be less than the LE of the youngest cohort, but greater than period LE which in turn is greater than $C A L$.

To find ACLE, we must know the ultimate life expectancy of all active cohorts, information not available for at least a century after the time in question. Yet the need for that cohort data is inescapable, since the purpose of the measure is to determine average cohort life expectancy. Three points should be made. First, ACLE provides a precise and conceptually clear measure of average cohort LE. When it can be calculated, it provides a "gold standard", i.e. a clear, conceptually based measure that best captures the idea of an average cohort life expectancy. When ACLE cannot be calculated, it identifies what needs to be estimated. Second, in the context of dynamic population models it can be examined under different mortality scenarios and its performance compared to that of alternative measures. Third, given the continuing interest in cohort mortality and future mortality trends, the ACLE standard helps to focus attention on how best to estimate future mortality and to assess the errors in such estimates.

To gain a better appreciation for how cohort LE, period LE, CAL and ACLE differ from each other, the following section examines their values in the context of models with changing mortality. 


\section{Cohort and period models of changing mortality}

Model populations provide a useful way to examine the sensitivity of aggregate measures to known, patterned changes in period and cohort mortality. To begin, we assume a simple Gompertz form for the force of mortality and write

$$
\mu(a, t)=\exp [A+b a-f(t)]
$$

where $\mathrm{f}(t)$ is a known function of time. To start with the simplest dynamic model, we assume that $\mathrm{f}(t)=0$ for $t \leq 0$, and $\mathrm{f}(t)=\mathrm{k}$ for $t>0$, where $\mathrm{k}$ can be any constant.

Figures 1a and $1 \mathrm{~b}$ show values of period and cohort $\mathrm{LE}, A C L E$, and $C A L$ for $\mathrm{k}=1$ and $\mathrm{k}=-1$. In the Gompertz model, we take $\mathrm{b}=.1$, the conventional value for the pace of mortality increase over age, and set $A=-11.2146608$ so $\mu(50,0)=0.002$, a reasonable value for a contemporary low mortality population. With $b=.1$, putting $k=1$ is equivalent to a mortality "set back" of 10 years, as a person aged $\mathrm{x}$ becomes subject to the mortality risk that characterized a person aged $\mathrm{x}-10$. Similarly, $\mathrm{k}=-1$ is equivalent to a mortality "set ahead" of 10 years.

Figure 1 shows that at time 0 , when mortality shifts, the cohort LE completes its gradual transition from the old to the new level, while the period LE abruptly moves from the old life expectancy at birth to the new. At $\mathrm{t}=0, A C L E$ has completed most, but not all of its transition. $C A L$ begins its rise at time 0 , when deaths depart from 1 , and is the last measure to complete the transition. 
Figure 1a: Cohort and period life expectancy, ACLE and CAL for a Gompertz mortality model with $A=-11.2146608, b=0.1$ and, beginning at time 0 , $\mathbf{k}=\mathbf{1}$

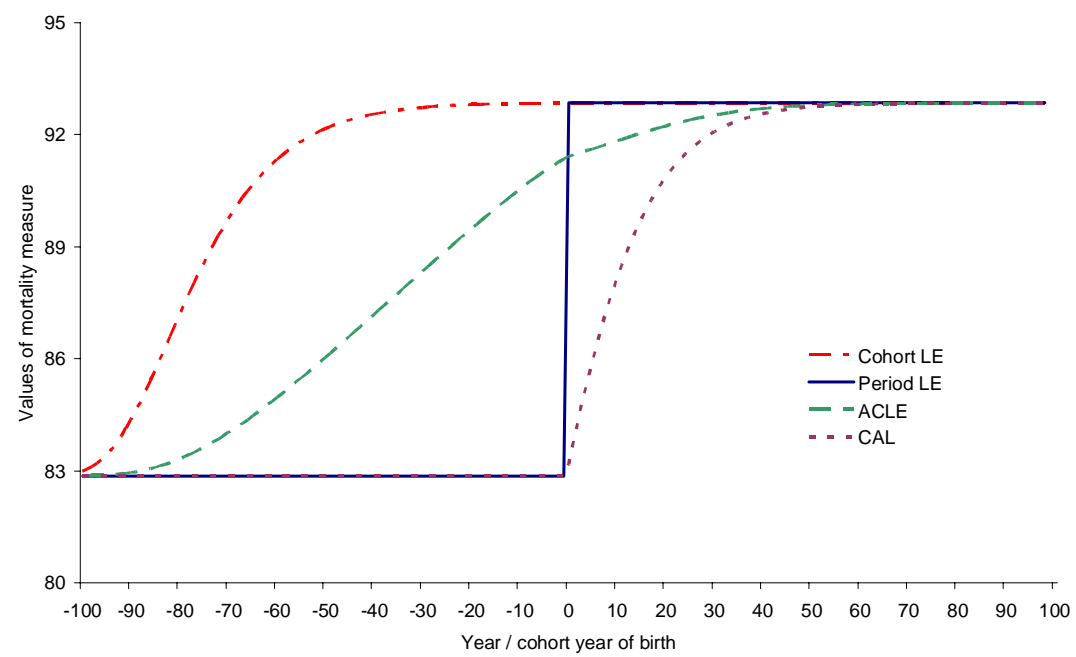

Figure 1b: Cohort and period life expectancy, ACLE and CAL for a Gompertz mortality model with $A=\mathbf{- 1 1 . 2 1 4 6 6 0 8 , ~} b=0.1$ and, beginning at time $0, k=-1$

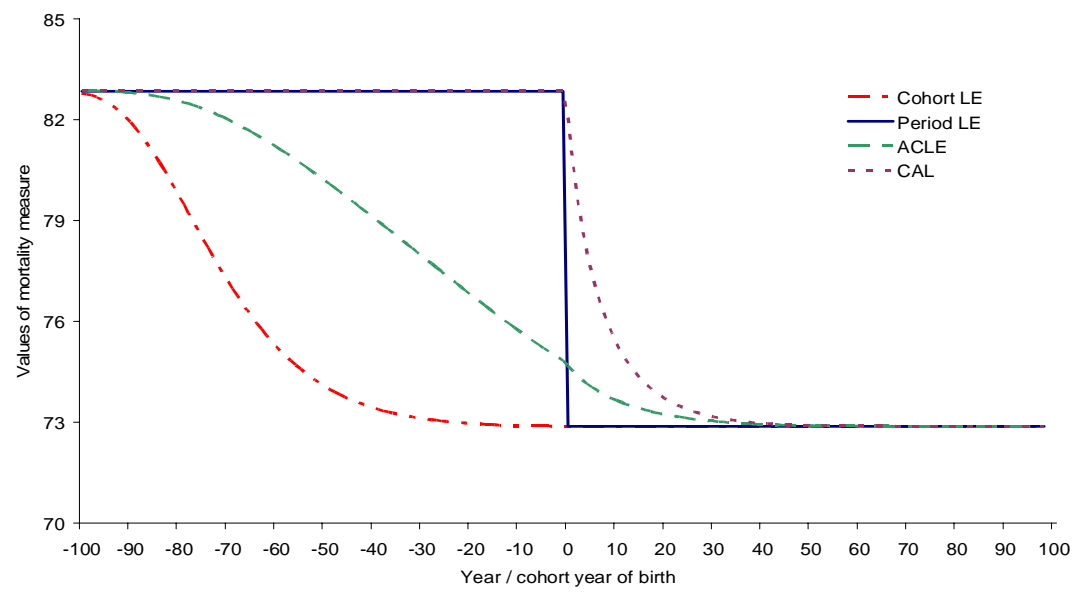


Figure 2a shows the total number of deaths occurring in the population each year, given one birth a year. Before time 0 , there is one death each year. At $\mathrm{t}=0$ and $\mathrm{k}=1$, there is an immediate fall to 0.38 deaths, with the annual number of deaths then increasing over time and eventually returning to one. Each year, $C A L$ grows by the amount that deaths are less than 1.

Wachter (2004) showed that $C A L(t)$ is a weighted average of period life expectancies for year $\mathrm{t}$ and preceding years. In this simple case, with only 2 period life expectancy values, it is possible to determine the weights involved. Specifically, we have

$$
\operatorname{CAL}(\mathrm{t})=\mathrm{w}(\mathrm{t}) \operatorname{LE}(-)+[1-\mathrm{w}(\mathrm{t})] \mathrm{LE}(+)
$$

Where $\mathrm{LE}(-)$ and $\mathrm{LE}(+)$ are the old and new life expectancies, respectively, and $\mathrm{w}(\mathrm{t})$ is the time $t$ fractional weight exerted by LE(-).

Figure $2 \mathbf{b}$ shows that after $t=0, w(t)$ declines to 0 in a roughly exponential fashion, with larger values of $\mathrm{k}$ associated with slower declines. The decline in $\mathrm{w}(\mathrm{t})$ need not be rapid. When $\mathrm{k}=1$ and $\mathrm{t}=20$, the rates prevailing 20 or more years earlier still exert a weight of 0.24 .

Let us now turn to an extension of the Gompertz model of mortality that traces a constant rate of decline over time. The basic model has been discussed by Vaupel (1986) and extended by Schoen, Jonsson and Tufis (2004). In that model, the force of mortality at age $a$ and time $t$ is defined as

$$
\mu(a, t)=e^{A+b a-c t},
$$

where A and $b$ retain their previously specified values. The annual rate of mortality improvement at all ages, $c$, is examined at two values: 0.008 , less than the recent rate of mortality decline in the West, and 0.015 , a closer approximation to many recent declines. 
Figure 2a: Total number of deaths in a population with one birth each year and Gompertz mortality shifting by $k$ at time 0

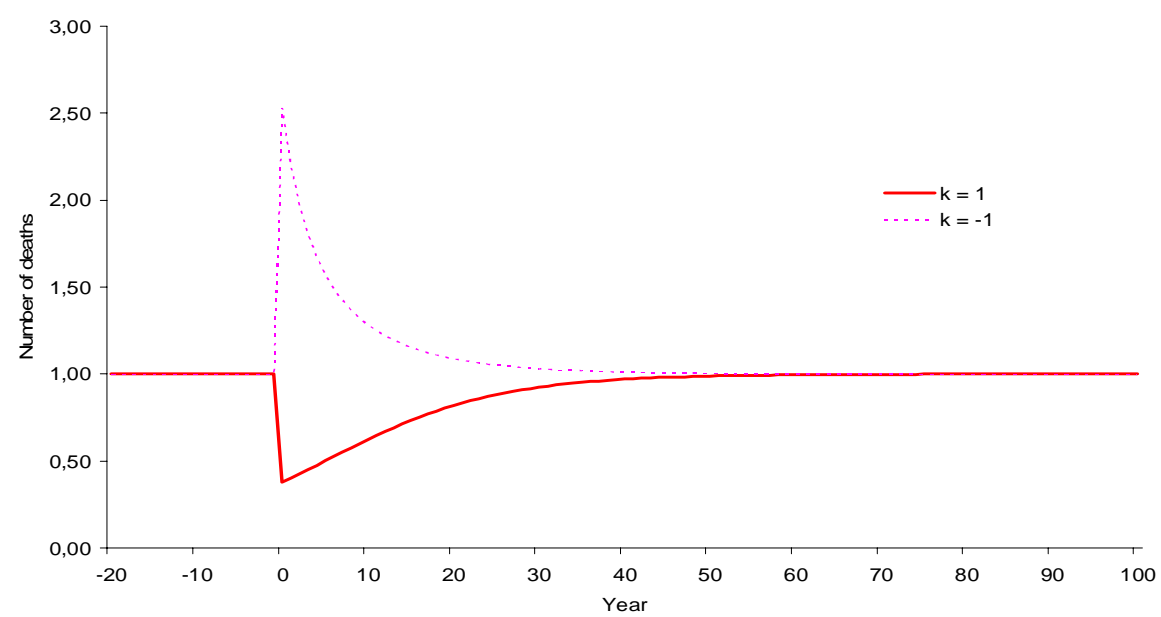

Figure 2b: Values of the weighting function $w(t)$ in the equation of CAL $(t)$ for a Gompertz mortality model with a shift in mortality by $\mathrm{k}$ at time 0; $\operatorname{CAL}(t)=w(t) \operatorname{LE}(-)+[1-w(t)] \operatorname{LE}(+)$

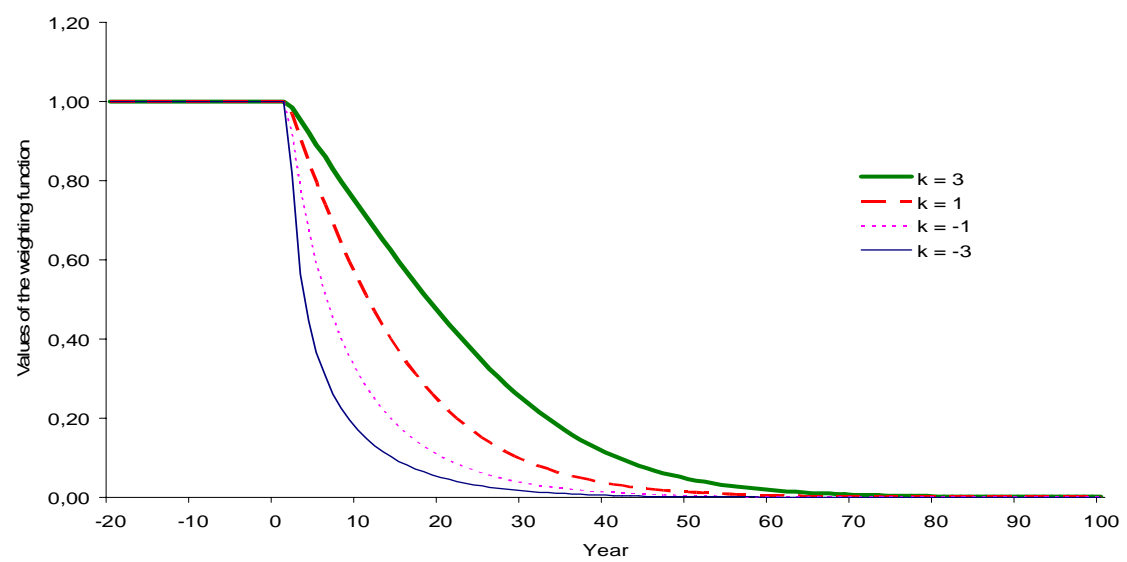


Figure 3 shows cohort and period LE, CAL, and ACLE for times 0 through 200 years based on equation (9) and our chosen parameter values for $A, b$, and $c$. All four measures are represented by virtually straight lines that appear to be parallel. At any given year $t$, the cohort life expectancy for those born in that year is the highest of the four measures, followed by $A C L E$, period life expectancy and $C A L$. The difference between $C A L$ and period life expectancy is particularly small (e.g. about 0.8 years for $\mathrm{c}=0.008$ ), and the LE/CAL ratio is about $1+\mathrm{c}$. The slope of the curves depends on parameters $\mathrm{b}$ and $\mathrm{c}$. For period LE and $C A L$, the slope is close to $\mathrm{c} / \mathrm{b}$. For cohort LE, it is about $\mathrm{c} /(\mathrm{b}-\mathrm{c})$, so over time the cohort LE line diverges from $C A L$ and period LE. $A C L E$ is roughly the arithmetic mean of the cohort and period life expectancies, and its slope approximates the average of the slopes of those expectancies. The ratio of ACLE to the cohort LE is close to constant over time, at a value of $1-c /(2 b)$.

Goldstein and Wachter (2005) studied gaps and lags between cohort and period life expectancies. They found that in populations with steady mortality declines the gap between cohort and period life expectancies at any point in time remains roughly constant, while the time lag increases. The time lag, $\lambda$, indicates the number of years between the time that cohort LE reaches a given level and the time period LE attains that level. In the model of Figure $3 \mathrm{a}, \lambda$ ranges from 74 years earlier at time 0 to 90 years earlier at time 200, while in the model of Figure $3 \mathrm{~b}$ those lag values are 74 and 103 years, respectively. Over those years, however, the gap only increases from 6.3 to 7.7 years in Figure 3a and from 12.8 to 18.1 years in Figure 3b.

Now, let us modify the mortality model in equation (9) to allow cyclical fluctuations over time. The new expression for the force of mortality can be written

$$
\mu(a, t)=e^{A+b a-(c t+d \sin (t \theta))},
$$

where $\theta=2 \pi / 60$ (about 0.10472 ) yields a cycle (from peak to peak) of 60 years, and $d=.25$ is a constant that moderates the amplitude of the oscillations. 
Figure 3a: Cohort and period life expectancy, ACLE, and CAL for a continually declining mortality model with $A=-\mathbf{1 1 . 2 1 4 6 6 0 8 , ~} b=0.1$ and $c=0.008$

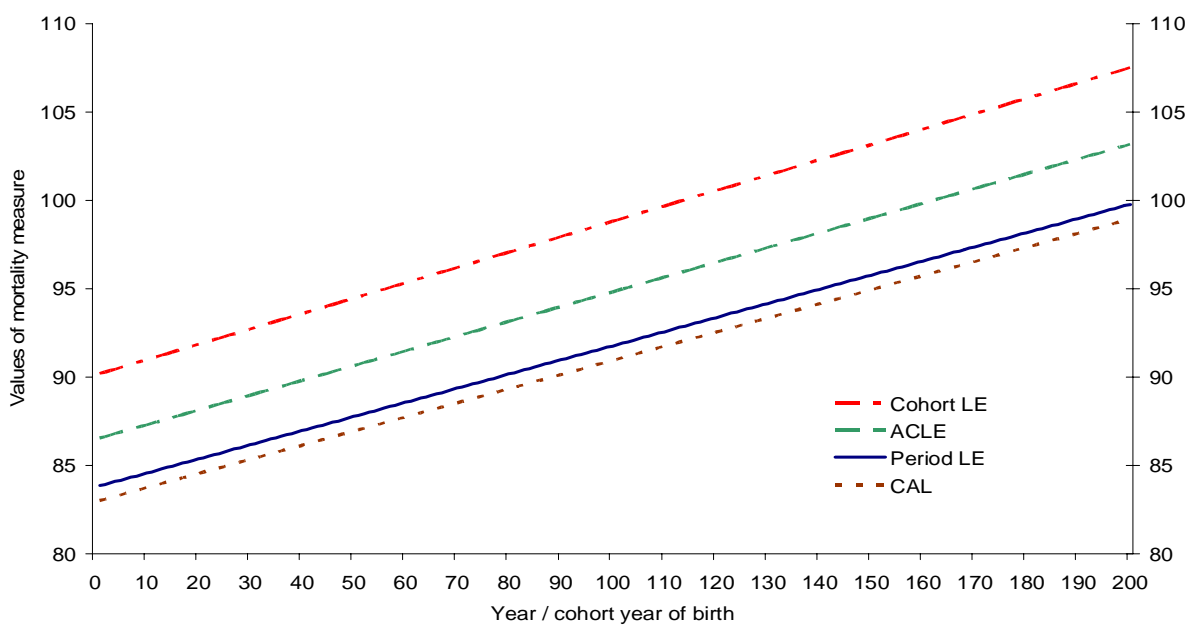

Figure 3b: Cohort and period life expectancy, ACLE, and CAL for a continually declining mortality model with $A=-\mathbf{1 1 . 2 1 4 6 6 0 8 , b = 0 . 1}$ and $\mathrm{c}=\mathbf{0 . 0 1 5}$

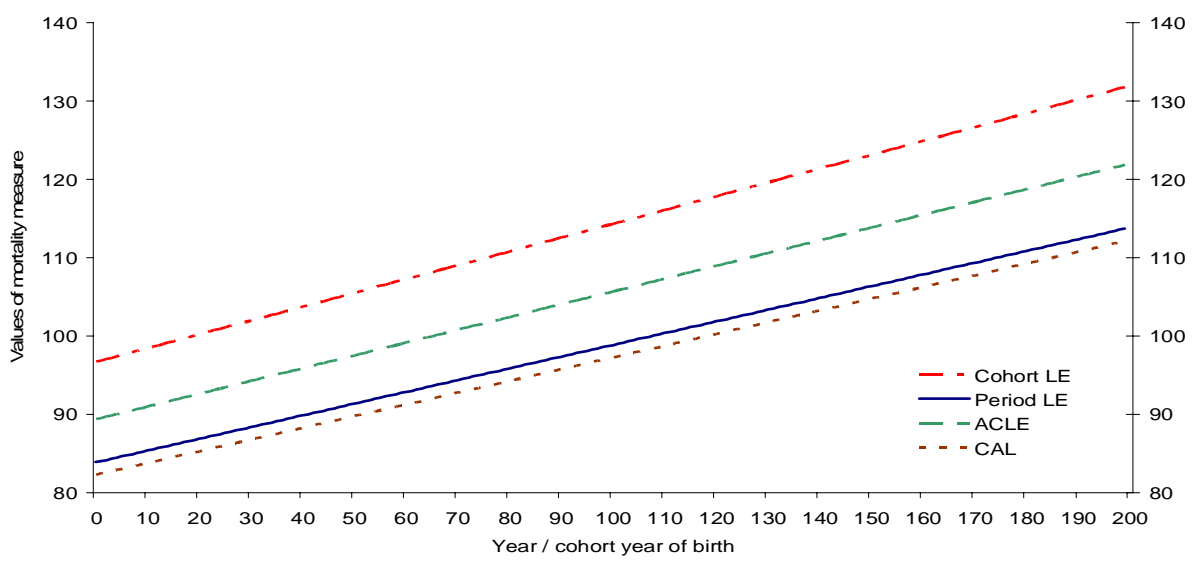


Figure 4a presents values of the cohort and period LE, CAL, and ACLE calculated on that basis. Those trajectories contrast markedly with those shown in Figure 3. The cohort LE now cycles modestly, though it is still the highest of the four measures. However, $C A L$ and the period LE alternate as the lowest measure, as they cycle with different phase shifts and the period LE has a slightly larger amplitude. The smoothest of the four measures is ACLE. As an average of cohort LEs, it cycles with an amplitude markedly smaller than that of the cohort LE.

The continually declining mortality model in Figure 3, which is based on equation (9), will yield similar results (though with a different rate of change over age) if rates change over cohorts instead of periods. That is not the case with Figure 4a, which is based on equation (10). To examine patterns when mortality changes cyclically over cohorts, we can write

$$
\mu(a, t)=e^{A+(b+c) a-(c t+d \sin ((t-a) \theta))} .
$$

The coefficient of the age variable is $(b+c)$ because age and time move together in the cohort perspective.

Figure 4b shows the results when cohort rates fluctuate sinusoidally as described by equation (11). The results are similar to those in Figure 4a, but here the cohort LE cycles with the largest amplitude. Period LE and $C A L$ alternate in the last position, though $C A L$ is usually lower. Again, $A C L E$ is the second highest measure and shows the smallest amount of fluctuation. 
Figure 4a: Cohort and period life expectancy, ACLE, and CAL for a sinusoidally declining mortality model with period cyclical fluctuations of 60 years and parameters $A=\mathbf{- 1 1 . 2 1 4 6 6 0 8 ,}$ $b=0.1$ and $c=0.008$

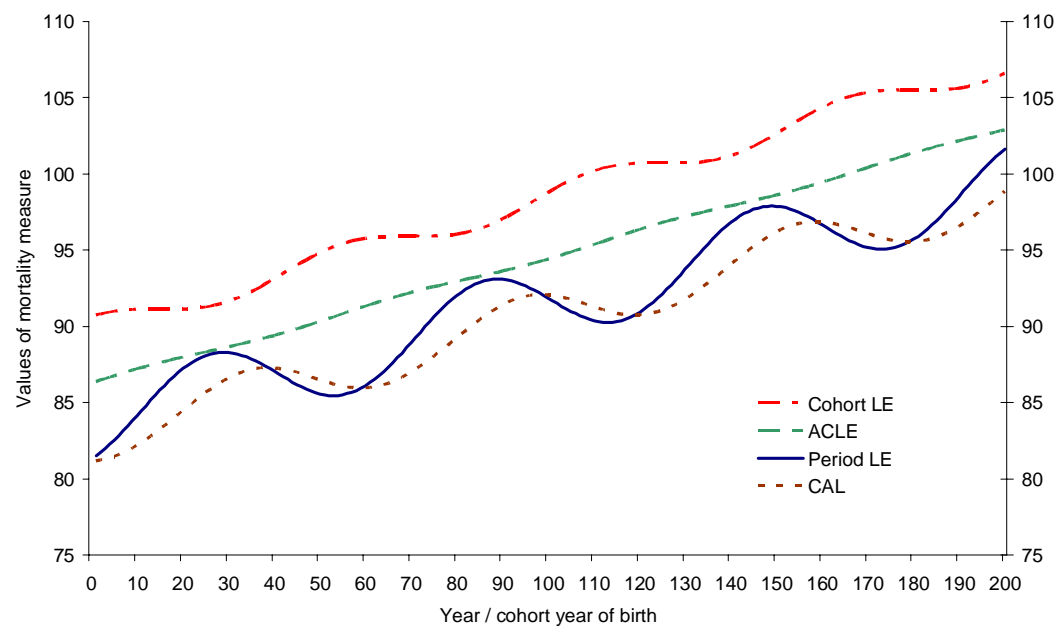

Figure 4b: Cohort and period life expectancy, ACLE, and CAL for a sinusoidally declining mortality model with cohort cyclical

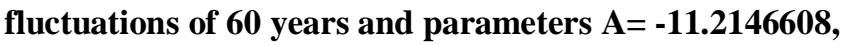
$b=0.1$ and $c=0.008$

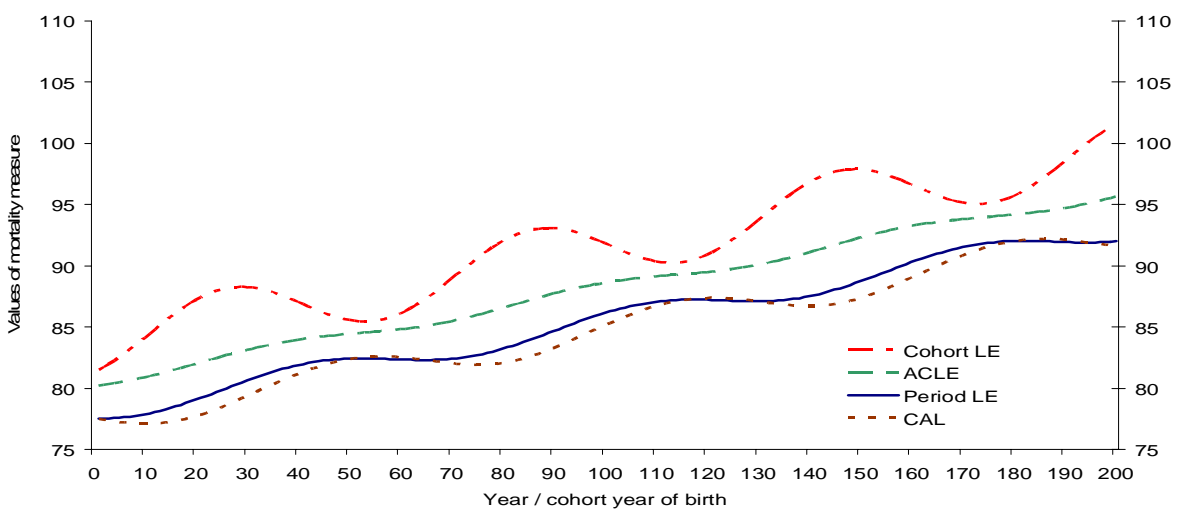


Guillot (2003b:20) suggested that tempo distortions in mortality be defined by adapting the approach used by Ryder (1964) in specifying timing effects in fertility. Accordingly, tempo effects on period measures are defined as those produced by changes in the timing of deaths within cohorts that do not affect the cohort LE. Following that definition, Figure 5 illustrates timing effects by introducing a tempo change, but no quantum change, in cohort mortality. The initial 25 and last 125 birth cohorts in those 200 cohorts are subject to the force of mortality

$$
\mu(a, t+a)=\exp [A+b a]
$$

where $A$ and $b$ have the same values as before. However, the cohorts born in years 26 through 75 have different parameters, specifically $A^{*}=10 \mathrm{e}^{A}=-8.91202$ and $b^{*}=0.067867$. As intended by that deliberate choice of $b^{*}$, those parameter changes increase mortality at younger ages, decrease mortality at older ages, but leave cohort LE fixed at 83.61 years for all cohorts.

Figure 5: Cohort and period life expectancy, ACLE, and CAL for a model with tempo changes in cohort mortality between years 25 and 75 but a fixed cohort LE

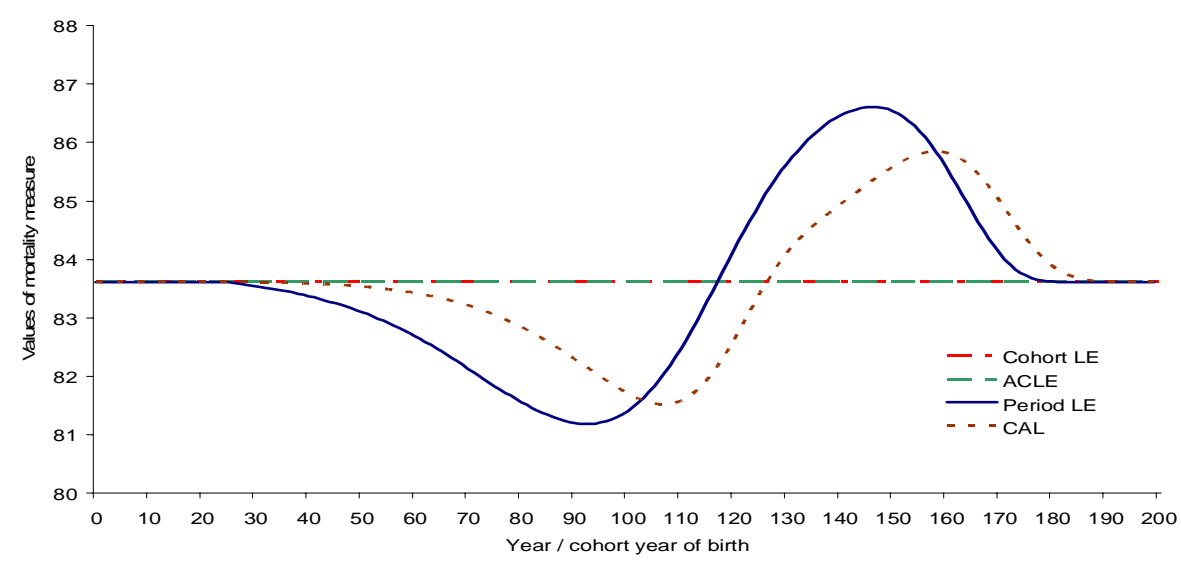


As a result, Figure 5 shows that cohort LE is a horizontal line. As evident from equations (6), a constant cohort LE produces ACLE values constant at that level. It follows that tempo distortions, as defined here, do not affect ACLE. However, those changes in timing substantially impact both the period LE and CAL. During years 80120, both period LE and $C A L$ decrease in size by more than 2, while during years 140170, both increase by more than 2. The fluctuation in $C A L$ was only a bit less than that in the period LE, indicating that in some circumstances $C A L$ is nearly as susceptible to tempo effects as is period life expectancy.

Mortality trends and fluctuations have long characterized human demographic history. As shown in the above models, under those conditions cohort LE, period LE, and $C A L$ can differ substantially from $A C L E$, the average life expectancy of the cohorts present in a given year. Next we examine those four measures of mortality in the context of three Western countries with a long history of data on mortality.

\section{ACLE in England and Wales, Norway, and Switzerland}

The following illustrations are based on data derived from the Human Mortality Database (2004). Estimates were made to calculate the average cohort life expectancy for years where full data on all active cohorts were not available. To complete cohort experience for years before the first available data year, we used the rates of the earliest available year. For cohorts not extinct by the latest data year, two scenarios were used. Scenario A completed cohort experience using death rates from the last available period. Scenario $B$ completed cohort experience by assuming that, at all ages, the death rates of the latest period declined by $c=0.005$ annually. Hence, if the last data year is 2000, then the calculations begin with the age-specific death rates for that year, $\mu(a, 2000)$. For age $a$ and for year $t>2000$, the force of mortality is given by

$$
\mu(a, t)=\mu(a, 2000) e^{-c(t-2000)} .
$$

Figures 6a, 6b, and $\mathbf{6 c}$ present the results for constant rate Scenario A, showing the cohort and period LE, CAL and ACLE for England and Wales, Norway and Switzerland. Similar patterns are observed in all three countries. The cohort LE has the highest value of all four aggregate measures. With the last available year used for future mortality, cohort and period LE converge to the same value. If that constant rate pattern continues, all four measures will converge. Initially, the period LE and ACLE alternate as the second largest value, with $C A L$ having the lowest value. More recently, as mortality declines continued, $A C L E$ has consistently been between the period LE and $C A L$. 
Figure 6a: Cohort and period life expectancy, ACLE, and CAL for England and Wales from 1880 to 1998 , with cohorts completed preserving the mortality observed in the last period

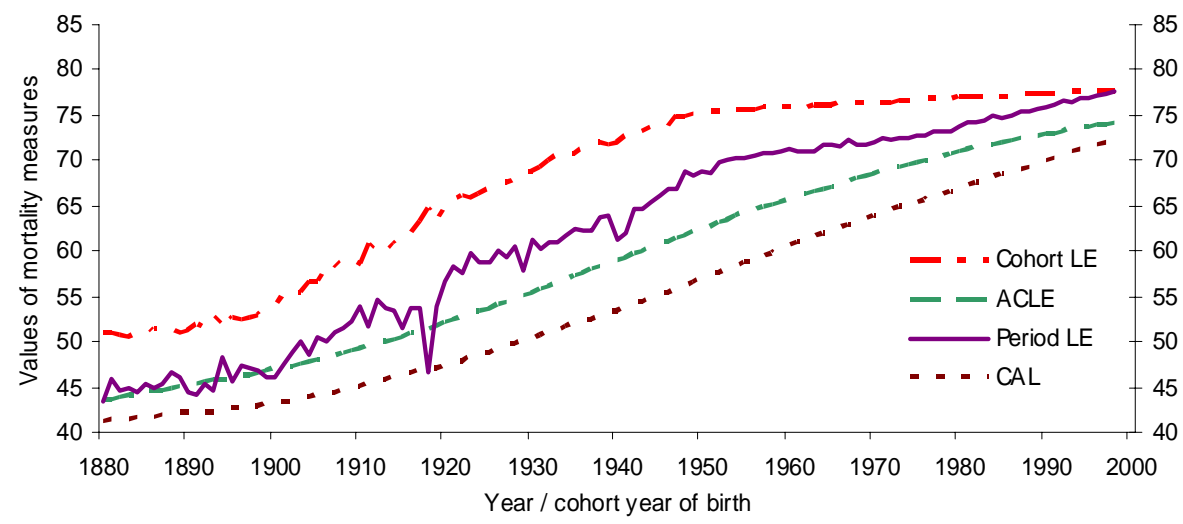

Figure 6b: Cohort and period life expectancy, ACLE, and CAL for Norway from 1880 to 2000, with cohorts completed preserving the mortality observed in the last period

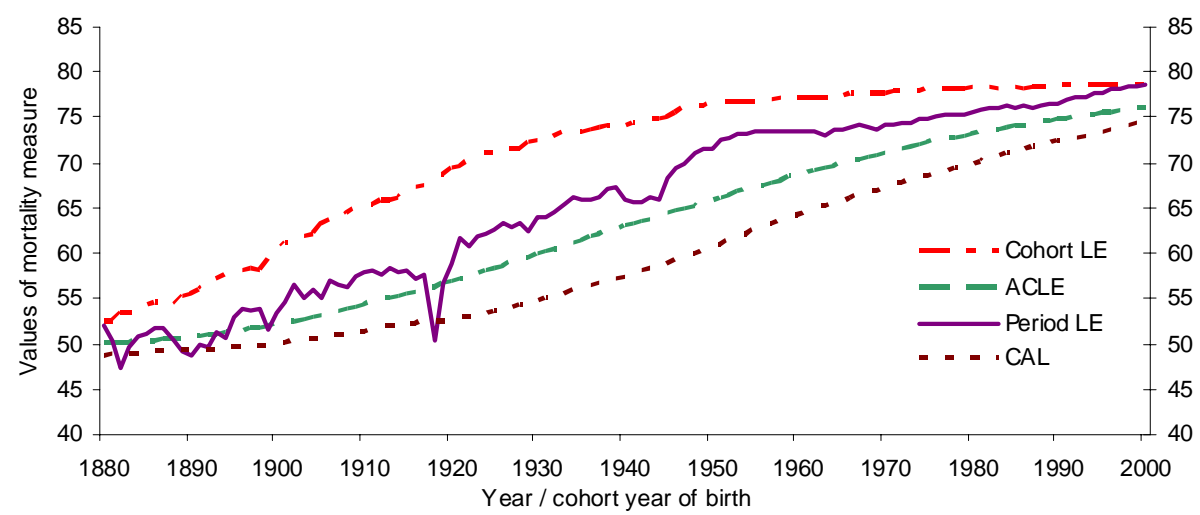


Figure 6c: Cohort and period life expectancy, ACLE, and CAL for Switzerland from 1880 to 2000 , with cohorts completed preserving the mortality observed in the last period

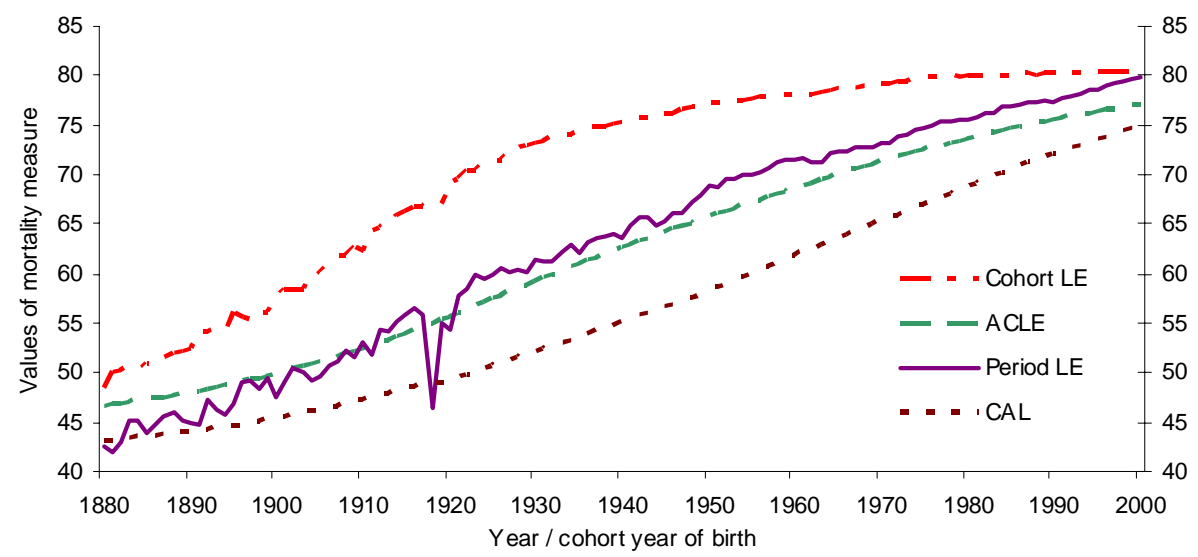

Figures 7a, 7b, and 7c present the results for declining future mortality Scenario B, showing our four measures for England and Wales, Norway, and Switzerland. With mortality continuing to improve at $0.5 \%$ per year, the cohort LE and ACLE show higher levels in Figure 7 than in Figure 6 from 1920 through the latest year shown. Consequently, ACLE exceeds the period LE during the last decades of the century. Differences between Figures 6 and 7 are fairly modest in all three countries. Nonetheless, with mortality steadily declining by more than $0.5 \%$ in recent decades, Figure 7 suggests that current life expectancies in many Western countries may understate the average life expectancy of living cohorts. 
Figure 7a: Cohort and period life expectancy, ACLE, and CAL for England and Wales from 1880 to 1998, cohorts completed by assuming a continually declining mortality model with $\mathrm{c}=\mathbf{0 . 0 0 5}$

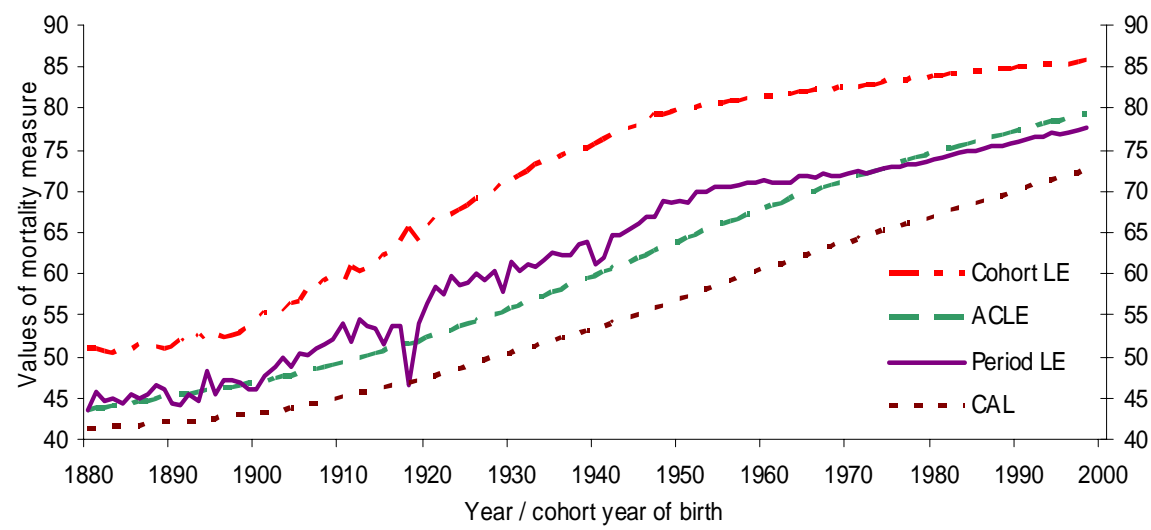

Figure 7b: Cohort and period life expectancy, ACLE, and CAL for Norway from 1880 to 2000 , cohorts completed by assuming a continually declining mortality model with $\mathrm{c}=\mathbf{0 . 0 0 5}$

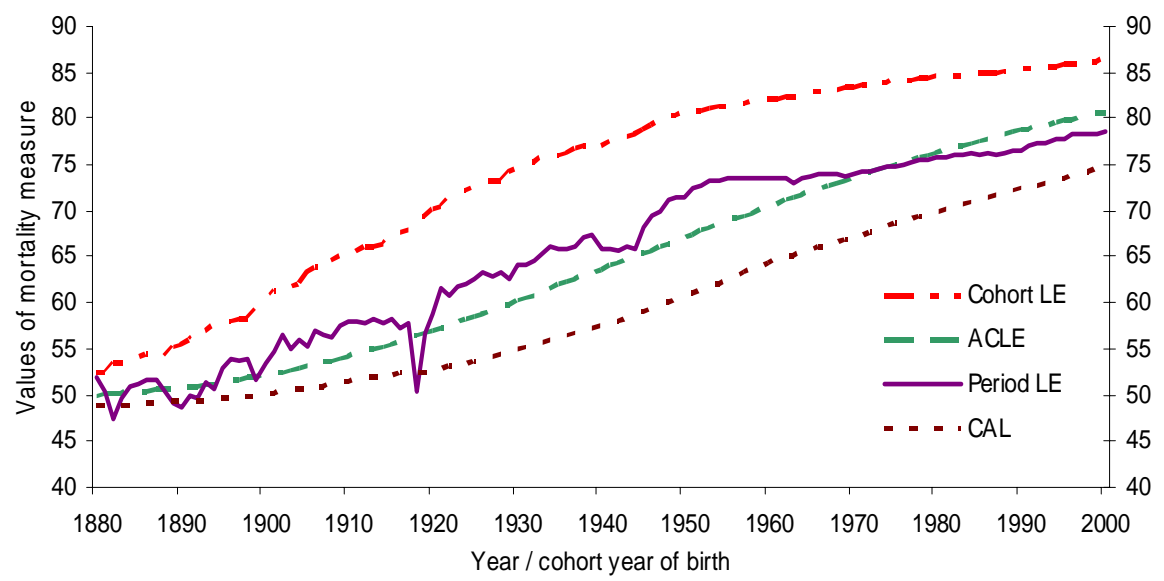


Figure 7c: Cohort and period life expectancy, ACLE, and CAL for Switzerland from 1880 to 2000. The cohorts have been completed following a continually declining mortality model with $\mathrm{c}=\mathbf{0 . 0 0 5}$

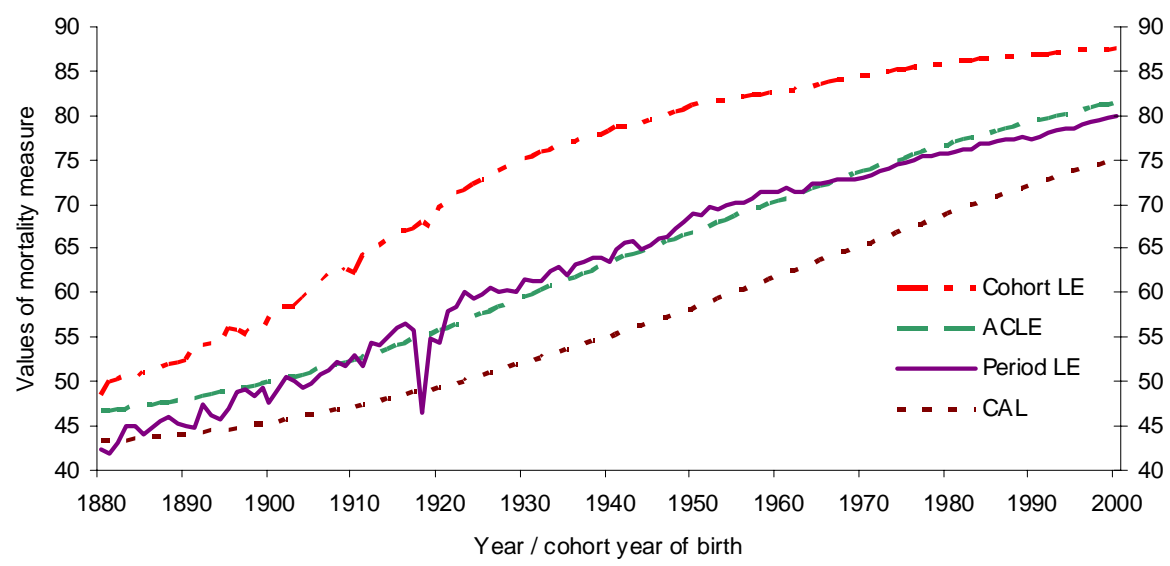

\section{Discussion and conclusions}

Recent discussions of mortality dynamics have noted that period life expectancies may not provide an accurate measure of the longevity of the population living in any given year. To provide such a measure, this paper presents $A C L E$, a new aggregate measure of survivorship. As derived, $A C L E$ is a weighted average of the life expectancies of the cohorts present in a given period, with each cohort weighted by its probability of survival to that given year.

Using population models with changing mortality and data from three Western European countries, we compared ACLE with period and cohort life expectancies and with $C A L$, a measure combining period and cohort mortality that has been advanced as an index of survivorship. $A C L E$ is relatively insensitive to both period and cohort fluctuations in death rates, and is unaffected by changes in the timing of mortality that do not change cohort life expectancy. In contrast, $C A L$ was found to be more sensitive to cohort and period fluctuations and to be quite susceptible to distortions from mortality timing effects. In models with continually declining mortality, and in contemporary Western populations with a long history of mortality declines that can reasonably be expected to continue, ACLE is larger than period life expectancy. 
However, $C A L$ is consistently less than period LE. If interpreted as a measure of tempo distortion, $C A L$ implies a downward level adjustment in the context of sustained increases in longevity.

The value of $A C L E$ is that it provides a quantitative, conceptually clear, and methodologically sound definition for the intuitive but vague idea of the average longevity of cohorts alive in a given year. ACLE demands data that span over two centuries, but those data are essential given what it seeks to measure. The necessary data are in fact available for a number of populations, and are fully knowable in the context of population models. Calculations for contemporary populations require estimates of the future course of mortality, but that is an active area of research and, provisionally, alternative scenarios can be used to assess the range of plausible outcomes.

Cohort LE only represents one cohort, period LE is sensitive to timing changes, and $C A L$ is not only sensitive to tempo effects but generally understates the period level of longevity. None of these problems are seen in ACLE, which reflects actual trends while minimizing fluctuations.

\section{Acknowledgements}

The second author gratefully acknowledges support from the DeWitt Wallace postdoctoral fellowship awarded by the Population Council. This is a revised version of the paper presented at the November 18-19, 2004 New York Workshop on Tempo Effects on Mortality sponsored by the Max Planck Institute for Demographic Research and the Population Council. 


\section{References}

Bongaarts, John. 2004. "Six period measures of longevity: A comment". Note presented at the Workshop on Tempo Effects on Mortality, New York, November 18-19.

Bongaarts, John and Griffith Feeney. 2002. "How long do we live?" Population and Development Review 28(1):13-29.

Brouard, Nicolas. 1986. "Structure et dynamique des populations. La pyramide des années à vivre, aspects nationaux et exemples régionaux," Espaces, Populations, Sociétés 2(14-15) : 157-68.

Canudas-Romo, Vladimir. 2003. Decomposition Methods in Demography. Rozenberg Publishers: Amsterdam, The Netherlands. Available at: www.ub.rug.nl/eldoc/dis/rw/v.canudas.romo/

Goldstein, Joshua R. and Kenneth W. Wachter. 2005. "Gaps and lags: Relationships between cohort and period life expectancy." Unpublished manuscript, Princeton University and University of California, Berkeley.

Guillot, Michel. 2003a. "The cross-sectional average length of life (CAL): A crosssectional mortality measure that reflects the experience of cohorts." Population Studies 57(1):41-54.

Guillot, Michel. 2003b. "Does period life expectancy overestimate current survival? An analysis of tempo effects in mortality." Paper presented at the PAA 2003 held in Minneapolis, Minnesota.

Human Mortality Database. University of California, Berkeley (USA), and Max Planck Institute for Demographic Research (Germany). Available at www.mortality.org or www.humanmortality.de (data downloaded on [5/1/04]).

Ryder, Norman B. 1964. "The process of demographic translation." Demography 1(1): 74-82.

Schoen, Robert, Stefan H. Jonsson and Paula Tufis. 2004. "A population with continually declining mortality." Working Paper 04-07, Population Research Institute, Pennsylvania State University, University Park.

Vaupel, James W. 1986. "How change in age-specific mortality affects life expectancy." Population Studies 40: 147-157. 
Vaupel, James W. 2002. "Life expectancy at current rates vs. current conditions: A reflexion stimulated by Bongaarts and Feeney's "How long do we live?"” Demographic Research 7: 365-378.

Vaupel, James W. and Vladimir Canudas-Romo. 2002. "Decomposing demographic change into direct vs. compositional components." Demographic Research 7: 114.

Wachter, Kenneth W. 2004. Tempo and its tribulations. Paper presented at the Workshop on Tempo Effects on Mortality, New York, November 18-19. 


\section{Appendix:}

\section{Decomposing the change over time in $A C L E$}

A further analysis of the dynamics of $A C L E$ and its decomposition show that typically the main component of change in this measure reflects changes in cohort life expectancies, not the survivorship weights used. The change over time in ACLE can be decomposed using the method introduced by Vaupel and Canudas-Romo (2002). It is possible to apply this decomposition because $A C L E(t)$ can be written as an average of cohort life expectancies weighted by cohort probabilities of survival, i.e. by

$$
\operatorname{ACLE}(t)=\frac{\int_{0}^{\omega} e_{c}(0, t-a) \ell_{c}(a, t-a) d a}{\int_{0}^{\omega} \ell_{c}(a, t-a) d a}=\overline{e_{c}}(t) .
$$

The change in this average is due to two components: first the average change in cohort life expectancies, or the direct change, and second changes in the distribution of the probabilities of survival, or the compositional change. Following the Vaupel and Canudas-Romo (2002) notation of a bar and dot over a variable to denote the average over age and the derivative over time, respectively, we obtain

$$
\frac{\partial A C L E}{\partial t}=\dot{\overline{e_{c}}}=\overline{e_{c}}+\operatorname{Cov}\left(e_{c}, r_{c}\right)
$$

where the average change in cohort life expectancies is expressed as

$$
\dot{e}_{c}(t)=\frac{\int_{0}^{\omega} \frac{\partial e_{c}(0, t-a)}{\partial t} \ell_{c}(a, t-a) d a}{\int_{0}^{\omega} \ell_{c}(a, t-a) d a} .
$$


The expression $r_{c}(a, t-a)$ denotes cohort age-specific growth rates, i.e. $r_{c}(a, t-a)=\frac{\partial \ln \left[\ell_{c}(a, t-a)\right]}{\partial t}$, and the covariance term is calculated as

$$
\operatorname{Cov}\left(e_{c}, r_{c}\right)=\frac{\int_{0}^{\omega} e_{c}(0, t-a) r_{c}(a, t-a) \ell_{c}(a, t-a) d a}{\int_{0}^{\omega} \ell_{c}(a, t-a) d a}-\operatorname{ACLE}(t) \frac{\int_{0}^{\omega} r_{c}(a, t-a) \ell_{c}(a, t-a) d a}{\int_{0}^{\omega} \ell_{c}(a, t-a) d a} .
$$

Table A-1: Change over time in ACLE and its decomposition: England and Wales

\begin{tabular}{ccccccc}
\hline $\begin{array}{c}\text { Year } \\
\mathrm{t}\end{array}$ & $\begin{array}{c}\mathrm{ACLE} \\
\mathrm{t}-5\end{array}$ & $\begin{array}{c}\text { ACLE } \\
\mathrm{t}+5\end{array}$ & $\begin{array}{c}\text { Change in } \\
\text { ACLE }\end{array}$ & $\begin{array}{c}\text { Average } \\
\text { change } \\
{[1]}\end{array}$ & $\begin{array}{c}\text { Covariance } \\
{[2]}\end{array}$ & $\begin{array}{c}\text { Equation } \\
(11)\end{array}$ \\
\hline 1905 & 46.891 & 49.283 & 0.239 & 0.246 & -0.007 & 0.239 \\
1915 & 49.283 & 52.234 & 0.295 & 0.304 & -0.009 & 0.295 \\
1925 & 52.234 & 55.864 & 0.363 & 0.375 & -0.012 & 0.363 \\
1935 & 55.864 & 59.866 & 0.400 & 0.418 & -0.018 & 0.400 \\
1945 & 59.866 & 63.925 & 0.406 & 0.434 & -0.028 & 0.406 \\
1955 & 63.925 & 67.891 & 0.397 & 0.425 & -0.028 & 0.397 \\
1965 & 67.891 & 71.457 & 0.357 & 0.392 & -0.036 & 0.356 \\
1975 & 71.457 & 74.654 & 0.320 & 0.357 & -0.038 & 0.320 \\
1985 & 74.654 & 77.394 & 0.274 & 0.316 & -0.042 & 0.274 \\
$1994^{*}$ & 77.394 & 79.251 & 0.232 & 0.271 & -0.039 & 0.232 \\
\hline
\end{tabular}

*The data only go to 1998 , therefore the last period is for eight instead of ten years 
Table A-2: Change over time in ACLE and its decomposition: Norway

\begin{tabular}{ccccccc}
\hline $\begin{array}{c}\text { Year } \\
\mathrm{t}\end{array}$ & $\begin{array}{c}\text { ACLE } \\
\mathrm{t}-5\end{array}$ & $\begin{array}{c}\text { ACLE } \\
\mathrm{t}+5\end{array}$ & $\begin{array}{c}\text { Change in } \\
\text { ACLE }\end{array}$ & $\begin{array}{c}\text { Average } \\
\text { change } \\
{[1]}\end{array}$ & $\begin{array}{c}\text { Covariance } \\
{[2]}\end{array}$ & $\begin{array}{c}(11) \\
{[1+2]}\end{array}$ \\
\hline 1905 & 51.943 & 54.216 & 0.227 & 0.226 & 0.001 & 0.227 \\
1915 & 54.216 & 56.965 & 0.275 & 0.274 & 0.001 & 0.275 \\
1925 & 56.965 & 60.244 & 0.328 & 0.330 & -0.002 & 0.328 \\
1935 & 60.244 & 63.716 & 0.347 & 0.357 & -0.010 & 0.347 \\
1945 & 63.716 & 67.110 & 0.339 & 0.368 & -0.029 & 0.339 \\
1955 & 67.110 & 70.466 & 0.336 & 0.368 & -0.032 & 0.336 \\
1965 & 70.466 & 73.606 & 0.314 & 0.342 & -0.028 & 0.314 \\
1975 & 73.606 & 76.371 & 0.277 & 0.308 & -0.031 & 0.276 \\
1985 & 76.371 & 78.722 & 0.235 & 0.264 & -0.029 & 0.235 \\
1995 & 78.722 & 80.712 & 0.199 & 0.221 & -0.022 & 0.199 \\
\hline
\end{tabular}

Table A-3: Change over time in ACLE and its decomposition: Switzerland

\begin{tabular}{ccccccc}
\hline $\begin{array}{c}\text { Year } \\
\mathrm{t}\end{array}$ & $\begin{array}{c}\text { ACLE } \\
\mathrm{t}-5\end{array}$ & $\begin{array}{c}\text { ACLE } \\
\mathrm{t}+5\end{array}$ & $\begin{array}{c}\text { Change in } \\
\text { ACLE }\end{array}$ & $\begin{array}{c}\text { Average } \\
\text { change } \\
{[1]}\end{array}$ & $\begin{array}{c}\text { Covariance } \\
{[2]}\end{array}$ & $\begin{array}{c}\text { Equation } \\
(11)\end{array}$ \\
\hline 1905 & 52.977 & 55.063 & 0.209 & 0.243 & -0.043 & 0.201 \\
1915 & 55.063 & 57.645 & 0.258 & 0.280 & -0.027 & 0.252 \\
1925 & 57.645 & 60.611 & 0.297 & 0.309 & -0.015 & 0.294 \\
1935 & 60.611 & 63.673 & 0.306 & 0.312 & -0.006 & 0.306 \\
1945 & 63.673 & 66.920 & 0.325 & 0.342 & -0.016 & 0.326 \\
1955 & 66.920 & 70.372 & 0.345 & 0.383 & -0.038 & 0.345 \\
1965 & 70.372 & 73.726 & 0.335 & 0.383 & -0.048 & 0.335 \\
1975 & 73.726 & 76.706 & 0.298 & 0.352 & -0.054 & 0.298 \\
1985 & 76.706 & 79.272 & 0.257 & 0.305 & -0.048 & 0.256 \\
1995 & 79.272 & 81.431 & 0.216 & 0.253 & -0.037 & 0.216 \\
\hline
\end{tabular}

Discrepancies between the change in ACLE and the results in the last column arise because discrete data over a 10-year period are used to approximate derivatives (see Canudas-Romo, 2003) 
Tables A-1 to 3 show the annual change in ACLE for each decade of the twentieth century, and its decomposition, for three countries: England and Wales, Norway and Switzerland. The first column shows time $t$, the midpoint of the 10 year interval examined. The next two columns give values of $A C L E$ at the beginning (time $t-5$ ) and the end $(\mathrm{t}+5)$ of the interval. Column four is the annual change in ACLE. That change is then decomposed into two components: the "average change" (or the "direct" change) and the "covariance" (or the "compositional" change). They are calculated from equations (16) and (17), respectively. Those two components sum to the figure shown in the last column, which gives the value that follows from equation (15). That final column does not exactly reproduce the "Change in ACLE" column because of inconsistencies between the continuous equations and the discrete data (for a discussion and techniques for minimizing discrepancies, see Canudas-Romo 2003).

For the three countries considered, Tables A-1 to 3 show that the direct change explains most of the change in average cohort life expectancies over time. The contribution of the compositional component is minor and almost invariably negative, partially offsetting the increase in ACLE.

The pre-eminence of the direct changes in cohort life expectancy over time and the relative unimportance of the weights used was confirmed by an analysis using an alternative weighting function. Instead of using cohort survival probabilities, period survival probabilities were used as weights and an average cohort survival measure, $A C S$, was created. The decomposition of the ACS reinforced the importance of the direct effect. Although $A C S$ gives results similar to $A C L E$, the rationale for using those weights was weaker, and the ACS was abandoned. 